\title{
Spatial Memory in Foraging Games
}

\author{
Bryan E. Kerster ${ }^{1}$, Theo Rhodes ${ }^{2}$, and Christopher T. Kello ${ }^{1}$ * \\ ${ }^{1}$ Cognitive and Information Sciences \\ University of California, Merced \\ ${ }^{2}$ Department of Psychology \\ State University of New York, Oswego
}

* Corresponding Author:

Professor Christopher Kello

Cognitive and Information Sciences

University of California, Merced

Merced, CA 95343

Phone: 209-233-1874

ckello@ucmerced.edu

Running Title: Foraging Video Game

Keywords: Foraging, spatial memory, search model, area-restricted search 


\begin{abstract}
$\underline{\text { Abstract }}$
Foraging and foraging-like processes are found in spatial navigation, memory, visual search, and many other search functions in human cognition and behavior. Foraging is commonly theorized using either random or correlated movements based on Lévy walks, or a series of decisions to remain or leave proximal areas known as "patches". Neither class of model makes use of spatial memory, but search performance may be enhanced when information about searched and unsearched locations is encoded. A video game was developed to test the role of human spatial memory in a canonical foraging task. Analyses of search trajectories from over 2000 human players yielded evidence that foraging movements were inherently clustered, and that clustering was facilitated by spatial memory cues and influenced by memory for spatial locations of targets found. A simple foraging model is presented in which spatial memory is used to integrate aspects of Lévy-based and patch-based foraging theories to perform a kind of area-restricted search, and thereby enhance performance as search unfolds. Using only two free parameters, the model accounts for a variety of findings that individually support competing theories, but together they argue for the integration of spatial memory into theories of foraging.
\end{abstract}




\section{Background}

Foraging is a class of general search processes often defined as one or more agents moving through a space to find targets that are relatively finite and have unknown locations. Examples of agents include humans, eyes, or algorithms, and minimization of movement is usually desirable to conserve energy, save time, and minimize risk. Foraging success is commonly defined as the rate at which targets are located per distance or time moved, and researchers have investigated how foraging may be optimized from different theoretical perspectives (Pyke, 1984; Viswanathan, da Luz, Raposo, \& Stanley, 2011).

Some researchers theorize foraging as random or correlated walks (Bartumeus, da Luz, Viswanathan, \& Catalan, 2005). Such stochastic processes do not require encoding of the environment, and their long-term behaviors may be determined analytically. Random walks with heavy-tailed path length distributions are better than those with normal distributions when targets are sparse and randomly distributed, because the resulting search trajectories cover more search area per unit time (Viswanathan, Buldyrev, Havlin, da Luz, Raposo, \& Stanley, 1999). Studies of animal foraging (Humphries, Weimerskirch, Queiroz, Southall, \& Sims, 2012), memory foraging (Rhodes \& Turvey, 2007), and visual foraging (Rhodes, Kello, \& Kerster, 2014) have all found path length distributions to be heavy-tailed, suggestive of some relationship between foraging and stochastic processes known as Lévy walks (Viswanathan, Afanasyev, Buldyrev, Murphy, Prince, \& Stanley, 1996). However, few if any search processes are literally Lévy walks because search dynamics are not purely stochastic, and this subtlety has led to much debate and contention about the role of random and correlated walks in theories of foraging (Pyke, 2015). 
Other researchers prefer to theorize foraging as an adaptive process that encodes information about the environment as search unfolds (Nathan, Getz, Revilla, Holyoak, Kadmon, Saltz, \& Smouse, 2008), in contrast with random and correlated walks. Encoding allows foraging movements to take advantage of environmental structure and regularities (Boyer \& Walsh, 2010). For instance, patch foraging (Charnov, 1976; Pirolli \& Card, 1999) is adaptive in that foraging decisions are based on ongoing estimates of instantaneous rates of target acquisition. The environment is divided into a set of local search regions (i.e., patches), and the fundamental task of search agents is to decide whether to continue foraging in the current patch, or leave to forage another patch. Under certain simplifying assumptions, the optimal decision rule is to stay as long as the current rate of target acquisition is above the long-term expected rate, and to leave when the current rate drops below the expectation (for applications to visual search, see Cain, Vul, Clark, \& Mitroff, 2012; Najemnik \& Geisler, 2005).

One simplifying assumption of random and correlated walks, as well as patch foraging, is to set aside the potential influence of spatial organization in the distribution of items being foraged. Area-restricted search processes (Hills, 2006; Kareiva \& Odell, 1987) are also adaptive and similar to patch foraging, but they are designed to capitalize on spatial clustering that often occurs in search environments. Search is concentrated near locations where targets are found by increasing the degree of turning (or decreasing the lengths of movements) as targets are found, and vice versa as targets are not found. Thus foraging movements remain relatively local when rates of acquisition are high, and become more long-ranging as rates decrease. It has been argued that area-restricted search is an evolutionary innovation (Hills \& Dukas, 2012), but the tradeoff relative to patch foraging is that area-restricted search has thus far been defined as heuristic, rather than optimal in terms of maximizing rates of target acquisition. 
Patch foraging and area-restricted search are adaptive processes in that they encode information about the environment as search unfolds, but this encoding is fairly minimal. Patch foraging only requires estimates of the current and long-term rates of target acquisition, and arearestricted search only requires an encoding of the current change in rate of target acquisition. Neither spatial memory nor distinct encodings for multiple targets are required. Evidence has been found supporting both patch foraging (Nonacs, 2001; Wolfe, 2003) and area-restricted search (see Hills, Todd, Lazer, Redish, \& Couzin, 2015), but it is clear that search processes can be enhanced by spatial memory (Boyer, Crofoot, \& Walsh, 2011; Fagan, Lewis, Auger-Méthé, Avgar, Benhamou, Breed, LaDage, Schlägel, Tang, Papastamatiou, Forester, \& Mueller, 2013), and there is evidence for the use of spatial memory in primate foraging (Janson \& Byrne, 2007; Janson, 1998).

When targets are finite and spatially fixed, spatial memory can help to minimize inefficient revisiting of previously searched locations. A number of visual search studies have investigated whether visual memory actually plays such a role, and while there has been some controversy (Wolfe \& Pokorny, 1990) and studies demonstrating a lack of memory (Horowitz \& Wolfe, 1998; Peterson, Kramer, Wang, Irwin, \& McCarley, 2001), it is clear that some amount of spatial memory is available to visual search (see Wolfe, 2003). Spatial memory can also be used to discern clustering and other spatial patterns to target locations that may be exploited. Therefore, it stands to reason that search processes will use spatial memory when available and not overly taxing on attentional, perceptual, or cognitive resources. However, it is difficult to test spatial memory in studies of naturalistic foraging, particularly in terms of manipulating factors hypothesized to affect foraging behaviors by virtue of spatial memory processes. 
To afford maximum experimental control over foraging conditions, we designed a video game to engage players in an abstraction of naturalistic foraging. Three aspects of the search environment were manipulated to test how spatial memory might guide search movements: Target density, target clustering, and spatial memory cues. Memory can be useful when targets are clustered because foraging should be drawn to clusters as they are located, and higher target densities should provide more information about the locations, shapes, and sizes of clusters. Results are presented and analyzed in terms of performance, path length distributions, spatial clustering, and dependency of search movements on targets found. We find evidence that search trajectories were inherently clustered, as Lévy walks would predict, but also that performance was enhanced by spatial memory —in particular, performance increased with visual landmarks and with increased spatial information in the form of greater target clustering and density. We present a new model of foraging that uses spatial memory to perform efficient search by embodying general properties of inherent clustering akin to Lévy foraging, and area-restricted search akin to patch foraging. We test the model against our search data, and discuss how the model may be adapted to a range of search processes in the behavioral and cognitive sciences.

\section{Methods}

A foraging video game (http://cogmech.ucmerced.edu/simple) was designed in which players moved a mouse cursor to search. Target locations were always hidden from view, and targets were not replenished once foraged. The foraging space had a maximum resolution of $1280 \times 1024$ target locations, and the space was scaled to the mouse and screen resolution available on each participant's computer. The cursor appeared as a pointer inside a $15 \times 15$ square outline that showed the area foraged when clicked. When a click uncovered one or more 
targets, a chime was sounded once for each target, with a maximum of 9 chimes in rapid succession. To motivate participants, the top 10 high scores were posted and shown to participants at the end of play.

Each participant completed two trials of 300 clicks each, preceded by practice rounds of 15 clicks. Each participant was randomly assigned to play the foraging game with or without visual landmarks. Visual landmarks were created by displaying one of six Hubble space images as a background. These landmarks could serve only as memory aids because target locations were independent of them, as participants were informed. A black background was shown when there were no visual landmarks. One of the two trials per player (chosen at random) included a small movement time cost: Rather than moving instantaneously from one click location to the next, the foraging square moved gradually across the screen at a rate of about 900 pixels per second. Participants had to wait until movement ended before clicking again to forage. Movement cost had no reliable effect on search, so these analyses are not reported or discussed further.

Each player also was randomly assigned to one of three target density conditions, crossed with one of three clustering conditions. There were 100 (sparse), 600 (medium), or 1100 (dense) targets available, and they were placed either randomly, or with one of two different degrees of stochastic clustering. Stochastic clustering was created by a probabilistic, recursive algorithm (Figure 2) with a parameter that could vary between random placement of targets with no clustering (0.5) and a single cluster of all targets placed in a single random location (0.0). Intermediate values created clusters recursively nested within clusters, randomly located and shaped, with varying degrees of dispersion. Parameter values of 0.1 (most clustered), 0.3 (less clustered), and 0.5 (random) were used for the experiment. 


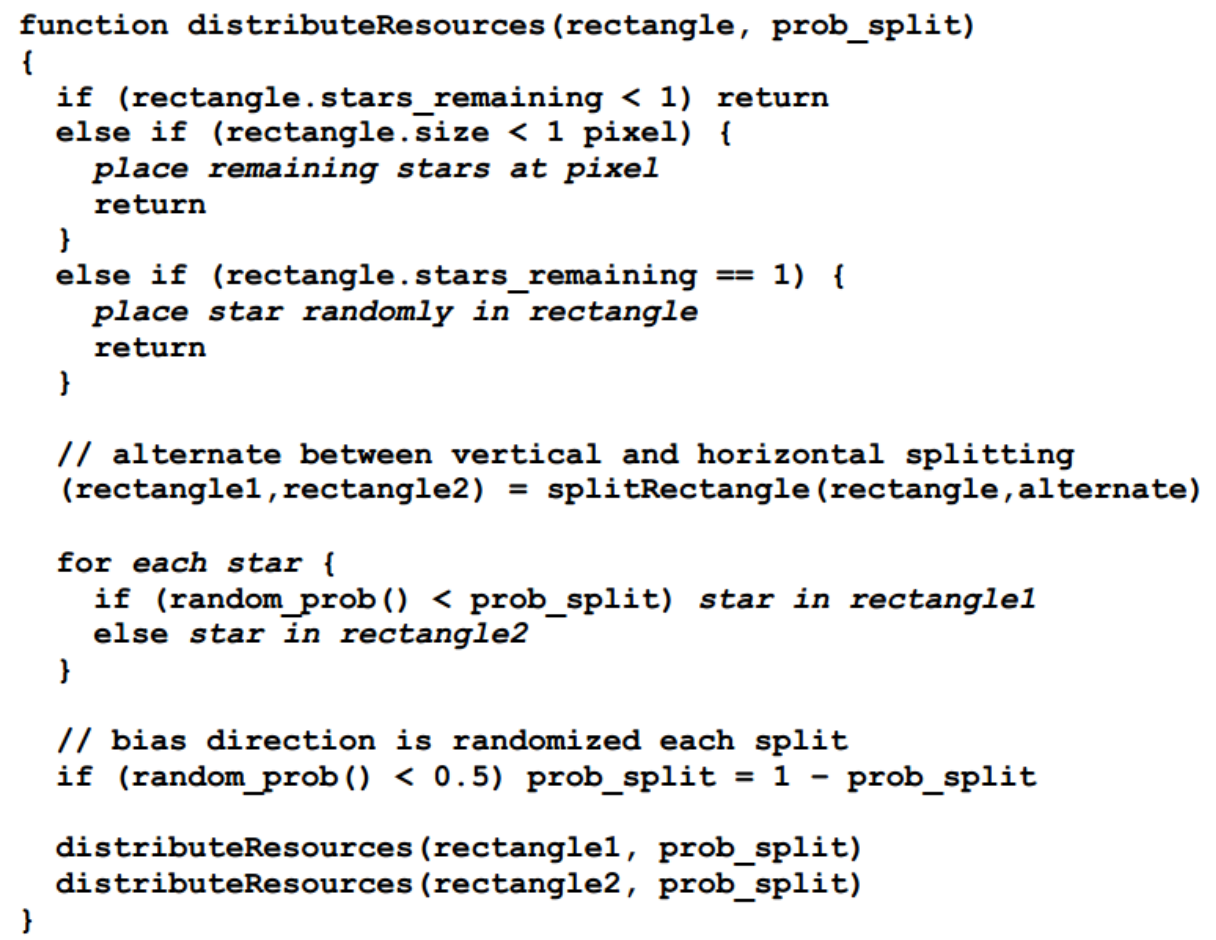

Figure 1. Pseudocode for recursive target clustering algorithm. The parameter prob_split determines the degree of clustering in generated target distributions, with 0.5 corresponding to a uniform random distribution, and 0 corresponding to all targets clustered at one random location. Intermediate values correspond to varying degrees of stochastic nested clustering.

Participants were recruited through Amazon's Mechanical Turk. A job was posted with the title "Space Foraging Game" and the description, "Play a fun game where you travel through space trying to find hidden resources. Takes about 5-10 minutes." The job was open for 2 days, during which over 2000 players with unique Amazon Turk IDs participated. Participants took 8 minutes on average to complete the job, and each player was paid 25 cents to complete the game. Nine players were excluded from analyses for failing to perform the task by clicking in the same location every time. Examples of paths produced by participants and background distributions can be viewed in lefthand column of Figure 2 . 

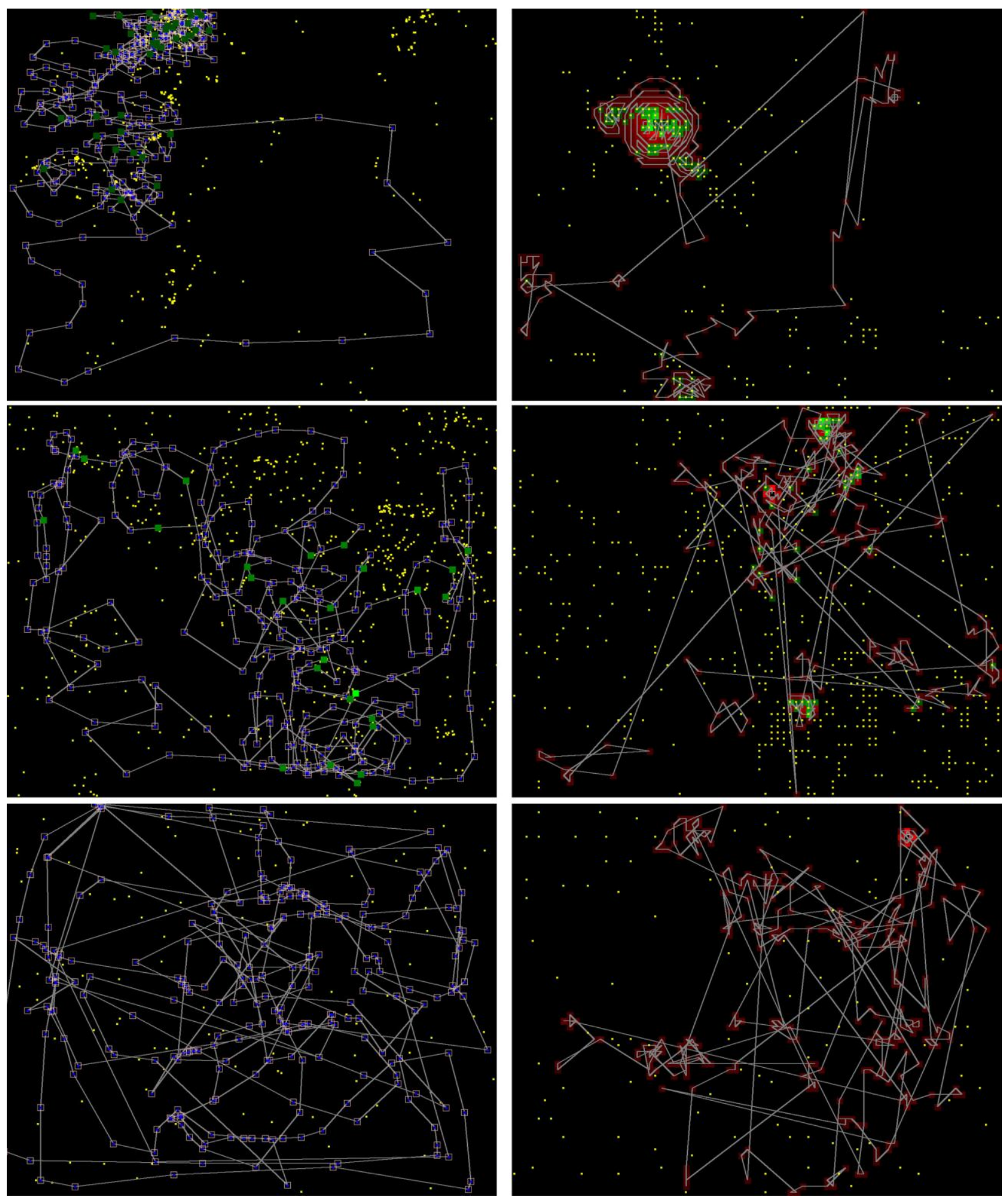

Figure 2. Example human (left) and model (right) foraging trajectories. Yellow dots show target locations (hidden to players), with brightness corresponding to number of targets. Squares 
show areas foraged for each click. For humans, a green square indicates that a resource was found. For the model, square color shows whether a positive (green) or negative (red) trace value is placed. Example resource distributions can be viewed in each image as well. The top row is high performance in the most clustered / high density resource condition, the middle row is average performance for less clustered / medium density resource condition, and the bottom row is low performance for randomly distributed / low density resource condition.

Participants were asked to identify the country in which they were currently located: 1519 identified the US, 406 identified India, 58 identified various other countries, and 11 declined to identify. Generalizing from the demographics of participants in previous studies on Mechanical Turk (Ross, Irani, Silberman, Zaldivar, \& Tomlinson, 2010), we estimate that the mean age of participants was about 31 , of which half were female.

\section{Results}

The Euclidean distance between each successive pair of clicks was calculated, and distances were integrated into summed path lengths for consecutive segments that formed an angle of less than $10^{\circ}$. We chose this threshold based on analyses of pilot search trajectories collected in the lab, for which we estimated the amount of imprecision and curvature in control of the computer mouse. We approximated $10^{\circ}$ to be one of the smaller angles that integrated over path deviations due to imprecision in mouse movements. We also found that variations in this threshold caused only minor changes in results, and no change in the main effects.

Each game yielded 165 integrated path lengths on average (i.e. with 300 clicks, each summed path was nearly two clicks long, on average). Path length distributions were analyzed along with percent targets found per player, as well as path lengths as a function of the number 
of consecutive locations foraged without targets. The effects of our foraging manipulations on these dependent measures were investigated to test how search trajectories vary as a function of target locations.

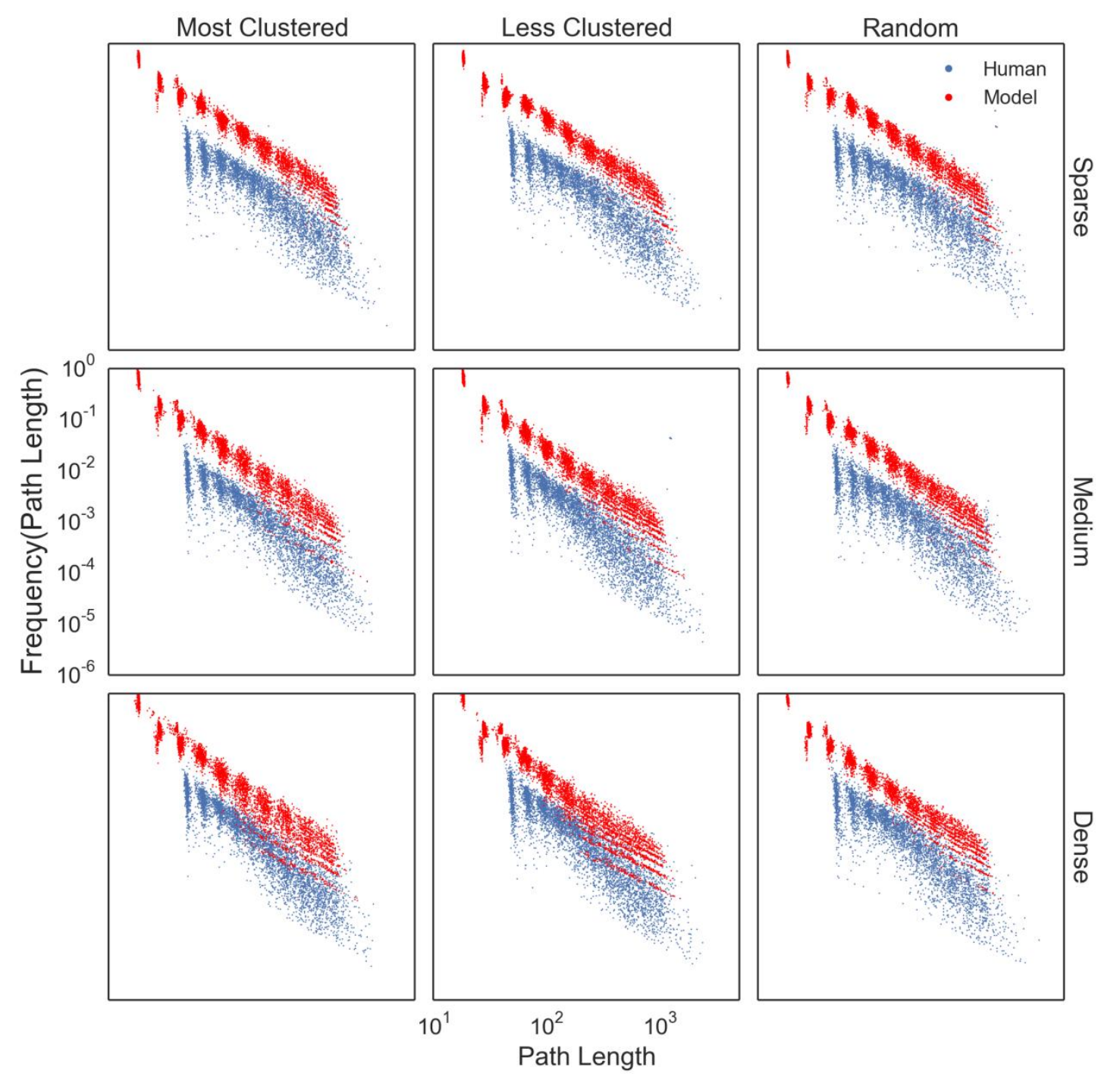

Figure 3. Path length distributions for human and model data. A histogram of 10

logarithmically-spaced bins was made for each game of 300 clicks, and all games are plotted together for each condition, separated by density and clustering (data with and without visual landmarks are combined). Path lengths less than 42 pixels are not plotted for humans because 
this was the empirically determined power law cutoff for observed path length distributions (see Methods section). Axis labels are shown only once, but all nine plots have the same axes.

Clustering of search trajectories was measured in two ways. First, path length distributions that can be quantified in terms of the functional forms of their tails-exponential, lognormal, and power law functions are most commonly used, the latter two being defined by tails heavier than exponential. Results using maximum likelihood methods suggest that foraging trajectories are sometimes best described by power laws or truncated power laws (Humphries, Queiroz, Dyer, Pade, Musyl, Schaefer, Fuller, Brunnschweiler, Doyle, Houghton, Hays, Jones, Noble, Wearmouth, Southall, \& Sims, 2010; Humphries et al., 2012), and other times by lognormal or stretched exponential functions (Breed, Severns, \& Edwards, 2015; Edwards, Freeman, Breed, \& Jonsen, 2012). All of these are heavy-tailed distributions and indicative of spatial clustering. Moreover, some researchers have argued that power law functions with exponents near two are indicative of Lévy walks which are optimal random walks under certain assumptions (Viswanathan et al., 1999), whereas others have criticized Lévy walks as overly simplistic models of foraging lacking in empirical support (Pyke, 2015).

For each path length distribution produced by each subject (Figure 3), Akaike's Information Criterion (AIC; Akaike, 1974) was applied to estimate the best fit of maximum likelihood estimates for the aforementioned three functions, plus truncated power law and Gaussian functions. The large majority of distributions were best fit by the lognormal (96\%), whose heavy tail is indicative of clustered search trajectories. While distributions were not strictly power law functions as predicted by Lévy walks, we could nonetheless estimate the amount of clustering by fitting a power law function to the tail of distribution. Tails were 
defined as path lengths $>42$ pixels, as determined using the method developed by Clauset and Shalizi (2009). Estimated exponents served to quantify each heavy tail (Rhee, Shin, Hong, Lee, Kim, \& Chong, 2011), and the cutoff separated out smaller path lengths that were more uniformly distributed, presumably due to noise from screen, mouse, and motor control limitations. Interestingly, estimated exponents were near the theoretic optimum of 2 for Lévy walks (Table 1), even though distributions were lognormal rather than power law.

Our finding of heavy-tailed (lognormal) path length distributions in all conditions indicates that search trajectories were inherently clustered. However, degrees of clustering, as measured by estimated exponents, changed as a function of targets distributions and visual landmarks. In particular, clustering of search trajectories increased with target density, decreased when targets were randomly distributed (Table 1), and increased with visual landmarks. These effects were tested using mixed effects analyses of variance (ANOVA) models, with subject as a random variable, visual landmarks and target density and clustering as independent variables, and estimated exponents and percent targets found as dependent variables.

Each effect was tested using likelihood ratios to compare the full mixed effects model against a null model that excluded the effect in question. With estimated exponent as the dependent variable, chi-square statistics showed reliable effects of target density, $\chi^{2}(2)=$ $90.18, p<.0001$ and target clustering, $\chi^{2}(2)=48.70, p<.0001$, and more clustering with visual landmarks $(M=1.92, \mathrm{SE}=0.007)$ than without $(\mathrm{M}=1.86, \mathrm{SE}=0.007), \chi^{2}(1)=18.54$, $p<.0001$. While these effects are clear and interpretable, we note that path length distributions are indirect measures of spatial clustering. A more direct method is first passage time analysis 
(Fauchald \& Tveraa, 2003), but it is not well-suited to measuring the nested clustering that is present in target distributions, and hypothesized in search trajectories.

The second way that we measured clustering in search trajectories was spatial Allan Factor analysis. Allan Factor analysis is tailored to measuring nested clustering in point processes (Allan, 1966), and it has been used to measure spatial clustering in eye movement trajectories during visual foraging (Rhodes et al., 2014). It operates on spatial distributions of points, without reference to paths between them, and so can be applied to distributions of foraged locations as well as the underlying targets. Thus we can compare clustering in search trajectories with clustering in the spatial target distributions being searched.

We applied spatial Allan Factor analysis to target distributions as well as foraged locations from the path length distribution analyses. Allan Factor analysis begins by tiling the foraging space with squares of side length $L$, and counting the number of foraged locations $N$ within each square $i$. Differences in counts between vertically and horizontally adjacent squares are averaged, and normalized by twice the mean count,

$$
A(L)=\frac{\left\langle\left(N_{i}-N_{i+1}\right)^{2}\right\rangle}{2\langle N\rangle} .
$$

The Allan Factor statistic $A(L)$ is a measure of spatial variance at a given scale $L$, and variances are computed over the range of available scales $L$, where $L$ is varied as a power of 2 . If foraged locations are randomly distributed (i.e. a Poisson distribution), then $A(L) \sim 1$ for all $L$. If locations are clustered across timescales, then $A(L)>1$ and increases with $L$. If clusters are hierarchically nested (i.e. fractal) across spatial scales, then $A(L) \sim L^{\alpha}$, where $\alpha>0$. This power law can be expressed as a linear relationship in log-log coordinates, $\log [A(L)] \sim \alpha \log [L]$. 
Results of Allan Factor analyses converged with those of path length distributions (see Table 1). Clustering was measured by fitting a regression line to each Allan Factor function for each game play in log-log coordinates. Slopes were reliably $>0$ and hence clustering was greater than chance in all conditions ( $\mathrm{p}<.0001$ in all cases). Furthermore, Allan Factor slopes patterned with estimated exponents for path length distributions: Once again, chi-square statistics showed reliable effects of target density, $\chi^{2}(2)=113.45, p<.0001$ and target clustering, $\chi^{2}(2)=248.11, p<.0001$, although there was no reliable effect of visual landmarks $(\mathrm{M}=0.272, \mathrm{SE}=0.004)$ compared to $(\mathrm{M}=0.266, \mathrm{SE}=0.004), \chi^{2}(1)=0.42$.

Table 1 also shows that the clustering parameter of the recursive algorithm for distributing targets had the desired effect as measured by Allan Factor analysis. Table 1 also shows that clustering increased with target density, which is expected given that greater numbers of targets help to overcome the stochastic (random) component of the algorithm. Moreover, one can see that clustering in human search locations followed the same pattern of differences across conditions as observed for target distributions, with two notable exceptions: Effects on spatial clustering were weaker for human search locations compared with target distributions, and the former were clustered even when target distributions were random and hence not clustered beyond chance (i.e. an Allan Factor slope of zero).

The effects of target density and clustering indicate that players modified search trajectories based on information in the foraging environment. Analyses of search performance indicate that these modifications were adaptive because effects with estimated exponents as the dependent variable corresponded to similar effects with percentages of targets found: Target density and clustering again had reliable effects with percent targets found as the dependent variable, $\chi^{2}(2)=79.44, p<.0001$ and $\chi^{2}(2)=575.8, p<.0001$, where performance 
increased with density and was lowest when target distribution was random. Also in line with exponents, performance was marginally better with visual landmarks $(\mathrm{M}=7.51 \%, \mathrm{SE}=0.19)$ than without $(\mathrm{M}=7.03 \%, \mathrm{SE}=0.17), \chi^{2}(1)=3.39, p=.066$.

These additional results indicate that clustering behavior in search trajectories adapted to clustering structure in target distributions in order to enhance performance. We examined the relationship between search and target clustering directly by dividing the range of observed exponent values into 10 bins with equal numbers of data points, and plotting the mean exponent of each bin against its corresponding mean percent targets found (Figure 4). Graphs show positive linear relationships that are steeper with greater target clustering, and no discernable effect of target density. Using linear regression, estimated exponents were reliably correlated with percent found at each of the three levels of target density, and in both clustered conditions $(\mathrm{p}<.0001$ in each case). There was no such relationship in the random condition because finding targets provided no information about locations of other targets.

Finally, correlations between search and target clustering provide indirect evidence that foraging movements were adapted as search unfolded and specific target clusters were found. We also tested the dependence of search movements on learned information about target distributions more directly by examining the relationship between each individual path length and the corresponding number of prior clicks without finding a target (Figure 5). These analyses showed that players made shorter movements soon after finding targets, which became increasingly longer as the number of consecutive unsuccessful clicks increased. Hills et al. (2013) found a similar result in that turning angles were greater after finding targets, but this effect only held when targets were clustered, whereas we found evidence for area-restricted search in all conditions. There were three differences compared with the present study that may 
be responsible for the discrepant effect: We measured path lengths instead of turning angles, we measured the effect of consecutive failed searches instead of one successful search, and we used a bird's eye view instead of a first-person view of the foraging environment.

a

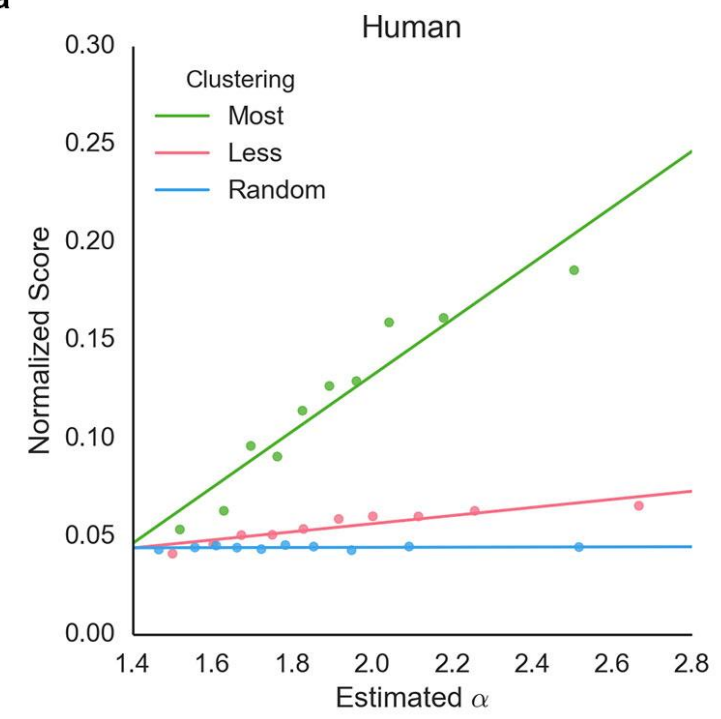

b

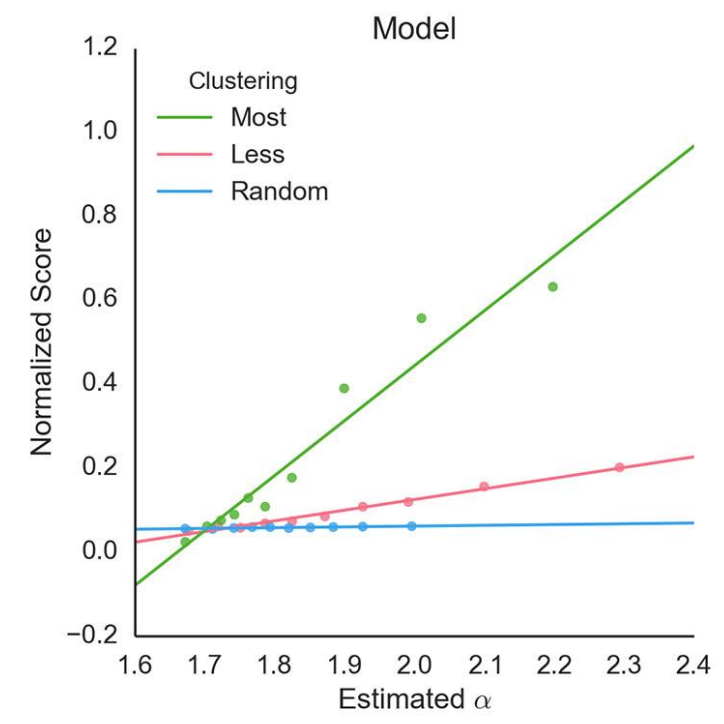

C

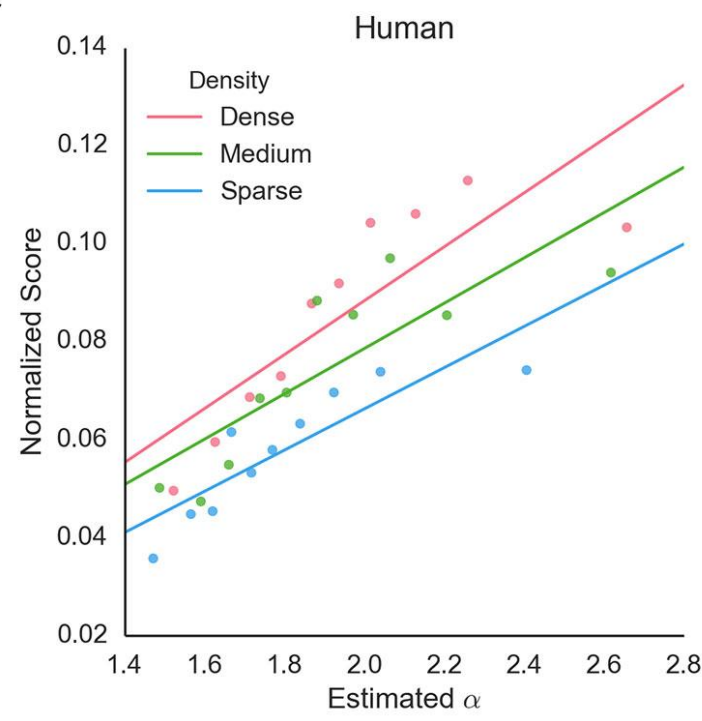

d

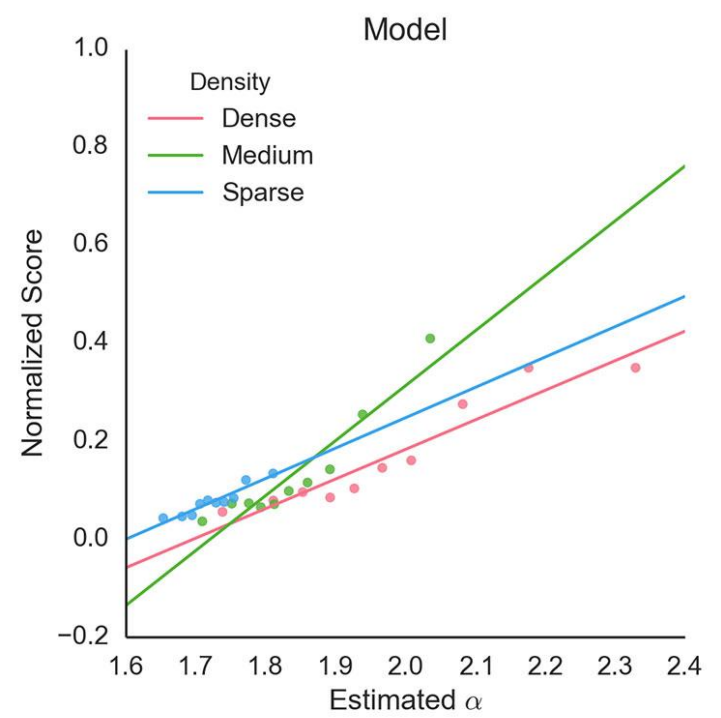

Figure 4. Relationship between \% found and estimated exponent. Positive linear

relationships are shown for $\%$ found and estimated exponent in the two clustered target 
conditions, for both human and model foraging trajectories. Target density is shown to have little or no effect on this relationship.
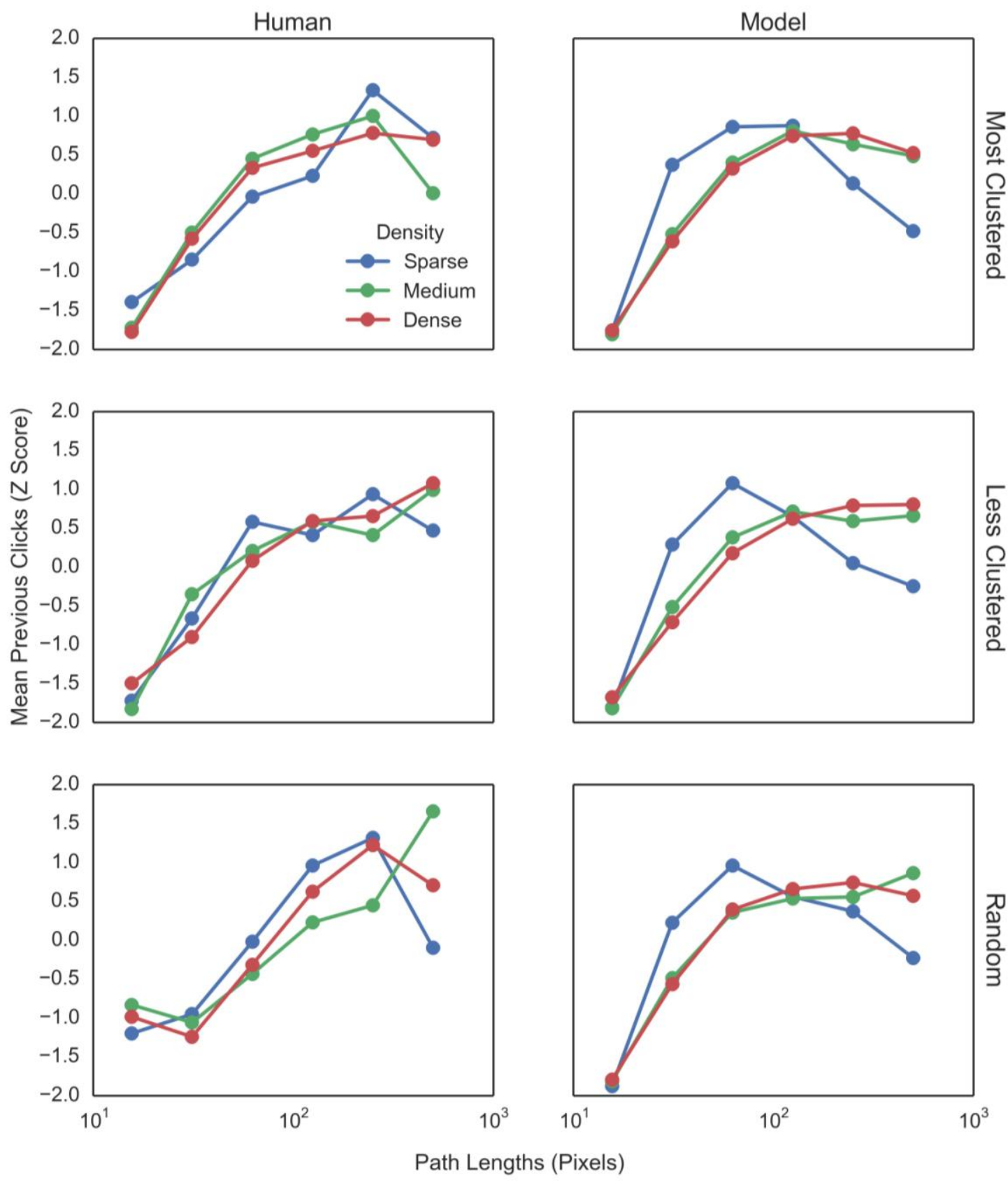

Figure 5. Area-restricted search behaviors. Path lengths are plotted against normalized Z scores of the mean number of previous consecutive clicks without finding a target, with path lengths logarithmically binned. Results are disaggregated by target density and clustering. Both 
human and model players show evidence of area-restricted search, with shorter path lengths sooner after finding targets.

\section{Spatial Memory Foraging Model}

Taken together, our results indicate that players searched more intensively near locations where targets were found, even when targets were randomly distributed, and they used spatial memory to aid this strategy. Players also showed a general bias towards clustered search, regardless of target distributions, and even when no targets were found (1.7\% of game plays yielded no targets, and of these $77.8 \%$ yielded heavy-tailed distributions).

To explain these results, we formulated a simple foraging model that was based on previous area-restricted search models (Hills, Kalff, \& Wiener, 2013), but included a spatial grid representation of expected target locations. The model was designed specifically to address three aspects of present and previous foraging data: 1) search movements are inherently clustered, regardless of foraging conditions (also see Rhodes et al., 2014); 2) search movements are adapted to clustering in target distributions; and 3) search movements are guided by spatial memory (also see Boyer \& Walsh, 2010; Fronhofer, Hovestadt, \& Poethke, 2013; Gautestad \& Mysterud, 2010). The model bears some resemblance to Gureckis and Markant's (2009) work on modeling active learning as applied to a spatial search game akin to Battleship. In both cases, information is uncovered as spatial locations are chosen efficiently based on their expected values. The main difference is that, in Gureckis and Markant (2009), expectations are derived from explicit hypotheses about three rectangles, whereas herein, expectations stem only from the particular locations of targets found, and a general bias towards clustering.

Our model assumes complete spatial and temporal memory as a matter of simplicity, rather than necessity — thus the observed memory benefits of visual landmarks fell outside the 
scope of the model. The foraging space is represented as 2D grid of squares, where the search agent chooses each move by sampling from a probability distribution over unvisited squares indexed by $x y$,

$$
\mathrm{P}\left[\exp \left(\gamma \frac{E_{x y}+1}{\beta M_{x y}+1}\right)\right]
$$

where $E_{x y}$ is the expected number of targets at square $x y, M_{x y}$ is the Euclidean distance from the agent's current location, and exponentials are normalized by their sum. The model has two free parameters, one to balance randomness versus determinism in selecting squares to visit $(\gamma)$, and one to weigh the effect of distance on movements $(\beta)$. The parameter space was searched by finding extremes for each parameter (beyond which there was little change in model performance), and then sampling the space within these extremes to find the best pair of values. The extremes were 2.5 and 8.5 for $\gamma$, and 0.05 and 0.6 for $\beta$, and the parameters were sampled in steps of 0.5 for $\gamma$ and 0.025 for $\beta$. The values of $\gamma=5.5$ and $\beta=0.125$ were found to approximately maximize overall fit of the model to the human data. These values were held constant across all simulations and conditions, which means that patterns of differential effects across conditions reflect how model behavior changes as a function of the target distribution and not because of changes in free parameters.

Expected numbers of targets are based on previously visited locations weighted by distance, i.e. $E_{x y}=\sum V_{i} / D_{i}$, where $V_{i}$ is the trace value at each previously visited square $i, D_{i}$ is the distance between $x y$ and each visited square, and the sum is over all previous visits. Traces encode numbers of targets as they are found, i.e. $V_{i}=N_{i}$ for $N>0$ upon visiting each square $i$. Thus expected numbers of targets are partly extrapolated from locations of previously found targets, and movements also are drawn to nearby locations. However, when no traces are found, 
an opposite, repelling force is applied, i.e. $V_{i}=-\ln \left(E_{x y}\right)$ for $N=0$. These negative values play an essential role in offsetting unmet expectations, and thereby driving movements away from previously found targets as searching around them fails to yield more targets (the logarithm attenuates repulsion and thereby ensures clustering). The dynamic can be conceptualized as a spatially continuous version of patch foraging: The model will tend to search locations near where targets were previously found (like an undefined patch), but as search repeatedly yields no further targets, $-\ln \left(E_{x y}\right)$ values will offset $N$ values and locations further away will be chosen instead (like exploring to find new patches). Spatial memory serves to guide movements towards or away from specific locations based on search history.

The model simulated each game play by each subject, and model trajectories were analyzed in the same manner as the human trajectories were. The model exhibited the same qualitative patterns of results as humans, shown throughout the figures and tables. Trajectories were inherently clustered with similar effects on Allan Factor slopes, albeit model search locations were overall more clustered than human search locations. Estimated exponents of path length distributions were very similar between human and model search trajectories, although model trajectories were all best fit by power law functions instead of lognormal. The power law means that model search trajectories resembled Lévy walks, which suggests that the model may help to explain the ubiquity of Lévy-like search trajectories in animal and cognitive foraging, as well as the effects of environmental conditions on Lévy walks in animal foraging (Ferreira, Raposo, Viswanathan, \& da Luz, 2012; Hills et al., 2013). The discrepancies with human players require further study, but may be due to the empirical limitations noted earlier.

Like humans, the model clustered movements around targets when first found, and then made longer movements away as searched locations were increasingly barren. This dynamic 
was an adaptive feature of the model, but the same underlying mechanism also caused inherent clustering even when no targets are found. The reason is that, when target expectations drop below one, $-\ln \left(E_{x y}\right)$ becomes positive and a trace is encoded as if a target was found. The search process then clusters around this internally generated trace, until enough empty locations are visited to trigger a long excursion, and the cycle may be repeated. This dynamic of target expectations was responsible for the intrinsic power law path length distributions, and it distinguished our model from previous foraging models with spatial memory (e.g. see Gautestad \& Mysterud, 2010).

Model performance and estimated exponents followed the same patterns as those observed for human players, including the variability in scores, as seen in similar coefficients of variation. The main differences between human and model foraging derived from the model's complete memory_-performance was about double that of humans when target distributions contained information about target locations, and path length distributions for the model were less variable than for human players, as seen in smaller coefficients of variation for estimated exponents.

\section{Discussion}

In the present study, evidence was found for the use of spatial memory in a simple foraging game designed to capture basic features of search processes found in numerous domains, including visual search (Klein \& MacInnes, 1999), memory search (Hills, Jones, \& Todd, 2012; Rhodes \& Turvey, 2007), and information search (Pirolli \& Card, 1999), as well as

foraging in animals and biological and artificial search agents in general. Players used spatial memory to take advantage of clustering in target distributions, akin to area-restricted search and patch foraging. However, search trajectories were clustered regardless of search conditions (cf. 
Scheibehenne, Wilke, \& Todd, 2011; Wilke \& Barrett), and path length distributions generally resembled those of Lévy walks (albeit distributions were best fit by lognormal functions rather than power laws).

We formulated a search model that used spatial memory to combine aspects of patch and Lévy foraging. Foraging movements are based on a spatial map that encodes targets found at foraged locations, as well as target expectations at not-yet-foraged locations. Expectations are derived from the combination of two opposing forces: Attraction towards locations near to where targets were previously found, and repulsion away from locations of where foraging was previously unsuccessful. Movements are probabilistically based on these two forces, combined with a cost on longer movement distances. Thus movements were biased towards maximizing a measure of search efficiency in environments for which targets are assumed to be clustered beyond chance.

Preliminary modeling work indicated that all three components just discussedattraction, repulsion, and movement cost—were necessary to account for human performance, as were the particular settings of the two free parameters. Model behavior changes in complex ways as the free parameters are varied, or when any of the components are altered or removed. However, we can identify three broad categories of deviations in model behavior relative to the human data (see Appendix for examples): 1) Movement locations can be too evenly distributed in space; 2) movement locations can produce multiple clusters that are each too densely clustered; or 3) movements can fail to produce a diverse range of clusters. The first and third categories are associated with exponential or Gaussian path length distributions instead of heavy tails, and the second category is associated with power law path length distributions that yield 
overly large estimated exponents. For all three categories of aberrant model behavior, search trajectories and performance fail to properly reflect the target distribution.

\subsection{Conclusions}

Our theoretical approach to foraging is akin to others that take advantage of environmental information (Boyer \& Walsh, 2010; Fagan et al., 2013; Fronhofer et al., 2013; Nabe-Nielsen, Tougaard, Teilmann, Lucke, \& Forchhammer, 2013). Our results showed that spatial memory was used to adapt movements to information about target clusters and thereby enhance performance. Our model contributes to work on spatial memory in domains like visual search (e.g. Elazary \& Itti, 2010) and navigation (Wang \& Spelke, 2002), as well as work on foraging. With respect to the latter, the model explains some patterns of behavior previously used to support patch foraging, and other patterns of behavior previously used to support foraging theories based on Lévy flights.

The validity of our study is based partly on the ability to generalize our results beyond foraging video games. Results are most likely to generalize to search processes in behavior and cognition that share properties with those examined in the present study. In particular, four major choices were made that define the class of search processes our work speaks to most directly, although it also may speak to other similar search processes as well.

The first choice was to make targets static and non-replenishing-each location had a fixed number of targets that were removed from the game when clicked. Some types of foraging in the animal kingdom are different in that targets can move (e.g. prey) or be replenished over time (e.g. nuts, fruits, and leaves). However, targets are often relatively static and nonreplenishing with respect to a single foraging episode — nuts and fruits take a long time to 
replenish and even longer to move location, for instance. Targets in perceptual and cognitive search tasks also are effectively fixed and non-replenishing in many cases, such as looking for places to hide from a given viewpoint, recalling a list of items or members of a semantic category, or manually searching for files on a hard drive (i.e. without keyword searching).

The second choice was to make the entire search space visually accessible, rather than limiting the field of view and requiring movement to access the entire space (see Wolbers \& Wiener, 2014). Some naturalistic foraging environments have limited fields of view, such as rodents searching through overgrown fields, or people searching through city streets. However, other search spaces are more readily accessible, e.g. for birds flying over a given hunting area, or people searching with the aid of maps. The entire space is generally available in visual search tasks, in that the search area is the field of view, and the fovea must be moved over specific locations to gain the visual resolution and processing necessary to identify targets. Comparisons with memory search tasks are less clear because the space is more abstract, but recent work using a whiteboard to spatialize a semantic search task indicates that parallels can be drawn between physical and mental search spaces (Montez, Thompson, \& Kello, 2015).

The third choice was to forego an investigation of long-term learning and adaptation to particular foraging conditions (Folse, Packard, \& Grant, 1989; Saarenmaa, Stone, Folse, Packard, Grant, Makela, \& Coulson, 1988). Human search processes are known to develop long-term adaptations for environmental regularities, particularly for spatial regularities such as the tendency for eye movements to scan the center of a scene and its horizontal meridian (Tatler, Hayhoe, Land, \& Ballard, 2011). In the present foraging game, for instance, participants may learn over time about the stochastic, nested nature of target clustering, and adapt their search processes to improve performance. 
The fourth and final choice was to forego any significant costs to foraging movements, either in terms of risk or time pressure or energy expenditure. The movement cost implemented turned out to be insufficient to affect search paths in any measurable way. Further experiments are needed to investigate the roles of learning and movement costs-for now, our study mainly speaks to the role of short-term spatial memory on foraging.

With regard to short-term spatial memory, this function is readily available in primates and other vertebrates with advanced neural structures such as the hippocampus (Suzuki \& Clayton, 2000). Spatial memory presumably is more limited than that of our model (Wang \& Spelke, 2002), but the model is simple enough and general enough that one could easily impose and test the effects of limited memory or perceptual capacities. One could impose limits on storage capacity or introduce decay, imprecision, or interference into memory traces, and one could limit the available foraging space to locations the search agent can immediately perceive or locomote to. We did not impose limits on memory or perception because doing so would entail additional free parameters, and because results show that these limits are not necessary to explain the pattern of results (except model performance was greater overall than human performance).

Finally, even for very simple search agents with little or no cognitive or memory capacities, spatial memory may be created externally as an emergent property of behavior, e.g. by leaving scent marks or other traces along search paths (Kamil \& Roitblat, 1985). The model is more abstract than specific mechanisms by which spatial memory may be implemented, and the process for determining foraging locations may be modified so that the trace values of locations are estimated and updated as movement unfolds. Thus the present data and model are intended to contribute to general theories of search based in spatial memory. 


\section{$\underline{\text { Acknowledgments }}$}

The authors thank Adrian Barr for his assistance with preliminary data collection, and Ajay Gopinathan and Anne Warlaumont for comments and feedback. This research was funded in part by a grant from the National Science Foundation, BCS 1031903. Foraging game data and code can be downloaded at http://cogmech.ucmerced.edu/downloads.html. 


\begin{tabular}{|c|c|c|c|c|c|c|c|c|c|c|c|c|c|}
\hline & \multicolumn{4}{|c|}{$\%$ Found } & \multicolumn{4}{|c|}{ Est. Exponent } & \multicolumn{5}{|c|}{ Allan Factor } \\
\hline & \multicolumn{2}{|c|}{$\underline{\text { Human }}$} & \multicolumn{2}{|c|}{$\underline{\text { Model }}$} & \multicolumn{2}{|c|}{$\underline{\text { Human }}$} & \multicolumn{2}{|c|}{$\underline{\text { Model }}$} & \multicolumn{2}{|c|}{$\underline{\text { Human }}$} & \multicolumn{2}{|c|}{ Model } & Targets \\
\hline & $9.9 \%$ & $(0.81)$ & $14.1 \%$ & $(1.00)$ & 1.84 & $(0.16)$ & 1.74 & $(0.03)$ & 0.24 & $(0.71)$ & 0.43 & $(0.11)$ & 0.27 \\
\hline Medium & $11.3 \%$ & $(0.90)$ & $22.5 \%$ & $(0.94)$ & 1.95 & $(0.19)$ & 1.89 & $(0.05)$ & 0.33 & $(0.59)$ & 0.52 & $(0.14)$ & 0.45 \\
\hline Dense & $13.2 \%$ & $(0.88)$ & $28.1 \%$ & $(0.80)$ & 2.00 & $(0.16)$ & 2.08 & $(0.08)$ & 0.38 & $(0.53)$ & 0.59 & $(0.13)$ & 0.51 \\
\hline$\underline{\text { Most }}$ & $21.7 \%$ & $(0.57)$ & $42.8 \%$ & $(0.53)$ & 1.97 & $(0.16)$ & 1.92 & $(0.09)$ & 0.41 & $(0.48)$ & 0.55 & $(0.17)$ & 0.76 \\
\hline Less & $7.4 \%$ & $(0.23)$ & $14.5 \%$ & $(0.35)$ & 2.00 & $(0.17)$ & 1.98 & $(0.11)$ & 0.34 & $(0.53)$ & 0.56 & $(0.13)$ & 0.44 \\
\hline Random & $5.5 \%$ & $(0.17)$ & $7.1 \%$ & $(0.17)$ & 1.81 & $(0.18)$ & 1.84 & $(0.05)$ & 0.19 & $(0.78)$ & 0.48 & $(0.10)$ & 0.03 \\
\hline
\end{tabular}

Table 1. Mean performance, estimated exponents, and Allan Factor results. Means for human and model results are shown along with coefficients of variation in parentheses, for percent correct, estimated exponents, and Allan Factor slopes (the latter also are shown for target distributions). Results for the top half of performances in each condition are shown to highlight the adaptive qualities of the best observed and simulated search behaviors. 


\section{$\underline{\text { References }}$}

Akaike, H. (1974). A new look at the statistical model identification. IEEE Transactions on Automatic Control, 19, 716-723.

Allan, D. W. (1966). Statistics of atomic frequency standards. Proceedings of the IEEE, 54(2), 221-230.

Bartumeus, F., da Luz, M. G. E., Viswanathan, G. M., \& Catalan, J. (2005). Animal search strategies: A quantitative random-walk analysis. Ecology, 86(11), 3078-3087.

Boyer, D., Crofoot, M. C., \& Walsh, P. D. (2011). Non-random walks in monkeys and humans. Journal of The Royal Society Interface.

Boyer, D., \& Walsh, P. D. (2010). Modelling the mobility of living organisms in heterogeneous landscapes: does memory improve foraging success? Philosophical Transactions of the Royal Society A: Mathematical, Physical and Engineering Sciences, 368(1933), 56455659.

Breed, G. A., Severns, P. M., \& Edwards, A. M. (2015). Apparent power-law distributions in animal movements can arise from intraspecific interactions. Journal of The Royal Society Interface, 12(103).

Cain, M. S., Vul, E., Clark, K., \& Mitroff, S. R. (2012). A Bayesian Optimal Foraging Model of Human Visual Search. Psychological Science.

Charnov, E. L. (1976). Optimal foraging, the marginal value theorem. Theoretical Population Biology, 9(2), 129-136.

Clauset, A., Shalizi, C. R., \& Newman, M. E. J. (2009). Power-law distributions in empirical data. SIAM Review, 51, 661-703. 
Edwards, A. M., Freeman, M. P., Breed, G. A., \& Jonsen, I. D. (2012). Incorrect Likelihood Methods Were Used to Infer Scaling Laws of Marine Predator Search Behaviour. PLoS ONE, 7(10), e45174.

Elazary, L., \& Itti, L. (2010). A Bayesian model for efficient visual search and recognition. Vision Research, 50(14), 1338-1352.

Fagan, W. F., Lewis, M. A., Auger-Méthé, M., Avgar, T., Benhamou, S., Breed, G., .. . Mueller, T. (2013). Spatial memory and animal movement. Ecology Letters, 16(10), 1316-1329.

Fauchald, P., \& Tveraa, T. (2003). Using first-passage time in the analysis of area-restricted search and habitat selection. Ecology, 84(2), 282-288.

Ferreira, A. S., Raposo, E. P., Viswanathan, G. M., \& da Luz, M. G. E. (2012). The influence of the environment on Lévy random search efficiency: Fractality and memory effects. Physica A: Statistical Mechanics and its Applications, 391(11), 3234-3246.

Folse, L. J., Packard, J. M., \& Grant, W. E. (1989). AI modelling of animal movements in a heterogeneous habitat. Ecological Modelling, 46(1-2), 57-72.

Fronhofer, E. A., Hovestadt, T., \& Poethke, H.-J. (2013). From random walks to informed movement. Oikos, 122(6), 857-866.

Gautestad, A. O., \& Mysterud, I. (2010). The home range fractal: From random walk to memorydependent space use. Ecological Complexity, 7(4), 458-470.

Gureckis, T. M., \& Markant, D. (2009). Active learning strategies in a spatial concept learning game Proceedings of the 31st Annual Conference of the Cognitive Science Society (pp. 3145-3150).

Hills, T. T. (2006). Animal foraging and the evolution of goal-directed cognition. Cognitive Science, 30(1), 3-41. 
Hills, T. T., \& Dukas, R. (2012). The evolution of cognitive search. Paper presented at the Cognitive Search: Evolution, Algorithms, and the Brain, Strüngmann Forum Report. Hills, T. T., Jones, M. N., \& Todd, P. M. (2012). Optimal foraging in semantic memory. Psychological Review, 119(2), 431-440.

Hills, T. T., Kalff, C., \& Wiener, J. M. (2013). Adaptive Lévy Processes and Area-Restricted Search in Human Foraging. PLoS ONE, 8(4), e60488.

Hills, T. T., Todd, P. M., Lazer, D., Redish, A. D., \& Couzin, I. D. (2015). Exploration versus exploitation in space, mind, and society. Trends in Cognitive Sciences, 19(1), 46-54.

Horowitz, T. S., \& Wolfe, J. M. (1998). Visual search has no memory. Nature, 394(6693), 575577.

Humphries, N. E., Queiroz, N., Dyer, J. R. M., Pade, N. G., Musyl, M. K., Schaefer, K. M., . . Sims, D. W. (2010). Environmental context explains Lévy and Brownian movement patterns of marine predators. Nature, 465(7301), 1066-1069.

Humphries, N. E., Weimerskirch, H., Queiroz, N., Southall, E. J., \& Sims, D. W. (2012). Foraging success of biological Lévy flights recorded in situ. Proceedings of the National Academy of Sciences, 109(19), 7169-7174.

Janson, C., \& Byrne, R. (2007). What wild primates know about resources: opening up the black box. Animal Cognition, 10(3), 357-367.

Janson, C. H. (1998). Experimental evidence for spatial memory in foraging wild capuchin monkeys, Cebus apella. Animal Behaviour, 55(5), 1229-1243.

Kamil, A. C., \& Roitblat, H. L. (1985). The ecology of foraging behavior: implications for animal learning and memory. Annual Review of Psychology, 36(1), 141-169. 
Kareiva, P., \& Odell, G. (1987). Swarms of predators exhibit" preytaxis" if individual predators use area-restricted search. American Naturalist, 130(2), 233-270.

Klein, R. M., \& MacInnes, W. J. (1999). Inhibition of Return is a Foraging Facilitator in Visual Search. Psychological Science, 10(4), 346-352.

Montez, P., Thompson, G., \& Kello, C. T. (2015). The Role of Semantic Clustering in Optimal Memory Foraging. Cognitive Science, n/a-n/a.

Nabe-Nielsen, J., Tougaard, J., Teilmann, J., Lucke, K., \& Forchhammer, M. C. (2013). How a simple adaptive foraging strategy can lead to emergent home ranges and increased food intake. Oikos, 122(9), 1307-1316.

Najemnik, J., \& Geisler, W. S. (2005). Optimal eye movement strategies in visual search. Nature, 434(7031), 387-391.

Nathan, R., Getz, W. M., Revilla, E., Holyoak, M., Kadmon, R., Saltz, D., \& Smouse, P. E. (2008). A movement ecology paradigm for unifying organismal movement research. Proceedings of the National Academy of Sciences, 105(49), 19052-19059.

Nonacs, P. (2001). State dependent behavior and the Marginal Value Theorem. Behavioral Ecology, 12(1), 71-83.

Peterson, M. S., Kramer, A. F., Wang, R. F., Irwin, D. E., \& McCarley, J. S. (2001). Visual Search has Memory. Psychological Science, 12(4), 287-292.

Pirolli, P., \& Card, S. (1999). Information foraging. Psychological Review, 106(4), 643-675.

Pyke, G. H. (1984). Optimal foraging theory: a critical review. Annual review of ecology and systematics, $15,523-575$.

Pyke, G. H. (2015). Understanding movements of organisms: it's time to abandon the Lévy foraging hypothesis. Methods in Ecology and Evolution, 6(1), 1-16. 
Rhee, I., Shin, M., Hong, S., Lee, K., Kim, S. J., \& Chong, S. (2011). On the levy-walk nature of human mobility. IEEE/ACM Trans. Netw., 19, 630-643.

Rhodes, T., Kello, C. T., \& Kerster, B. (2014). Intrinsic and extrinsic contributions to heavy tails in visual foraging. Visual Cognition, 22(6), 809-842.

Rhodes, T., \& Turvey, M. T. (2007). Human memory retrieval as Lévy foraging. Physica A, 385, 255-260.

Ross, J., Irani, L., Silberman, M. S., Zaldivar, A., \& Tomlinson, B. (2010). Who are the crowdworkers?: shifting demographics in mechanical turk. Paper presented at the CHI '10 Extended Abstracts on Human Factors in Computing Systems, Atlanta, Georgia, USA.

Saarenmaa, H., Stone, N. D., Folse, L. J., Packard, J. M., Grant, W. E., Makela, M. E., \& Coulson, R. N. (1988). An artificial intelligence modelling approach to simulating animal/habitat interactions. Ecological Modelling, 44(1-2), 125-141.

Scheibehenne, B., Wilke, A., \& Todd, P. M. (2011). Expectations of clumpy resources influence predictions of sequential events. Evolution and Human Behavior, 32(5), 326-333.

Suzuki, W. A., \& Clayton, N. S. (2000). The hippocampus and memory: a comparative and ethological perspective. Current Opinion in Neurobiology, 10(6), 768-773.

Tatler, B. W., Hayhoe, M. M., Land, M. F., \& Ballard, D. H. (2011). Eye guidance in natural vision: Reinterpreting salience. Journal of Vision, 11(5).

Viswanathan, G. M., Afanasyev, V., Buldyrev, S. V., Murphy, E. J., Prince, P. A., \& Stanley, H. E. (1996). Levy flight search patterns of wandering albatrosses. Nature, 381(6581), 413415. 
Viswanathan, G. M., Buldyrev, S. V., Havlin, S., da Luz, M. G. E., Raposo, E. P., \& Stanley, H. E. (1999). Optimizing the success of random searches. Nature, 401(6756), 911-914.

Viswanathan, G. M., da Luz, M. G. E., Raposo, E. P., \& Stanley, H. E. (2011). The physics of foraging: an introduction to random searches and biological encounters: Cambridge University Press Cambridge.

Wang, R. F., \& Spelke, E. S. (2002). Human spatial representation: insights from animals. Trends in Cognitive Sciences, 6(9), 376-382.

Wilke, A., \& Barrett, H. C. The hot hand phenomenon as a cognitive adaptation to clumped resources. Evolution and Human Behavior, 30(3), 161-169.

Wolbers, T., \& Wiener, J. M. (2014). Challenges for identifying the neural mechanisms that support spatial navigation: the impact of spatial scale. Frontiers in Human Neuroscience, 8,571 .

Wolfe, J., \& Pokorny, C. (1990). Inhibitory tagging in visual search: A failure to replicate. Perception \& Psychophysics, 48(4), 357-362.

Wolfe, J. M. (2003). Moving towards solutions to some enduring controversies in visual search. Trends in Cognitive Sciences, 7(2), 70-76. 


\section{Appendix}

The model has two free parameters, $\gamma$ and $\beta$, and thus four combinations of extreme values that lead to aberrant model behaviors. When $\gamma$ is very low, $\beta$ has no appreciable effect on model behavior, which means there are actually only three types of aberrant behaviors at the parameter extremes. These types are demonstrated in the following three figures.

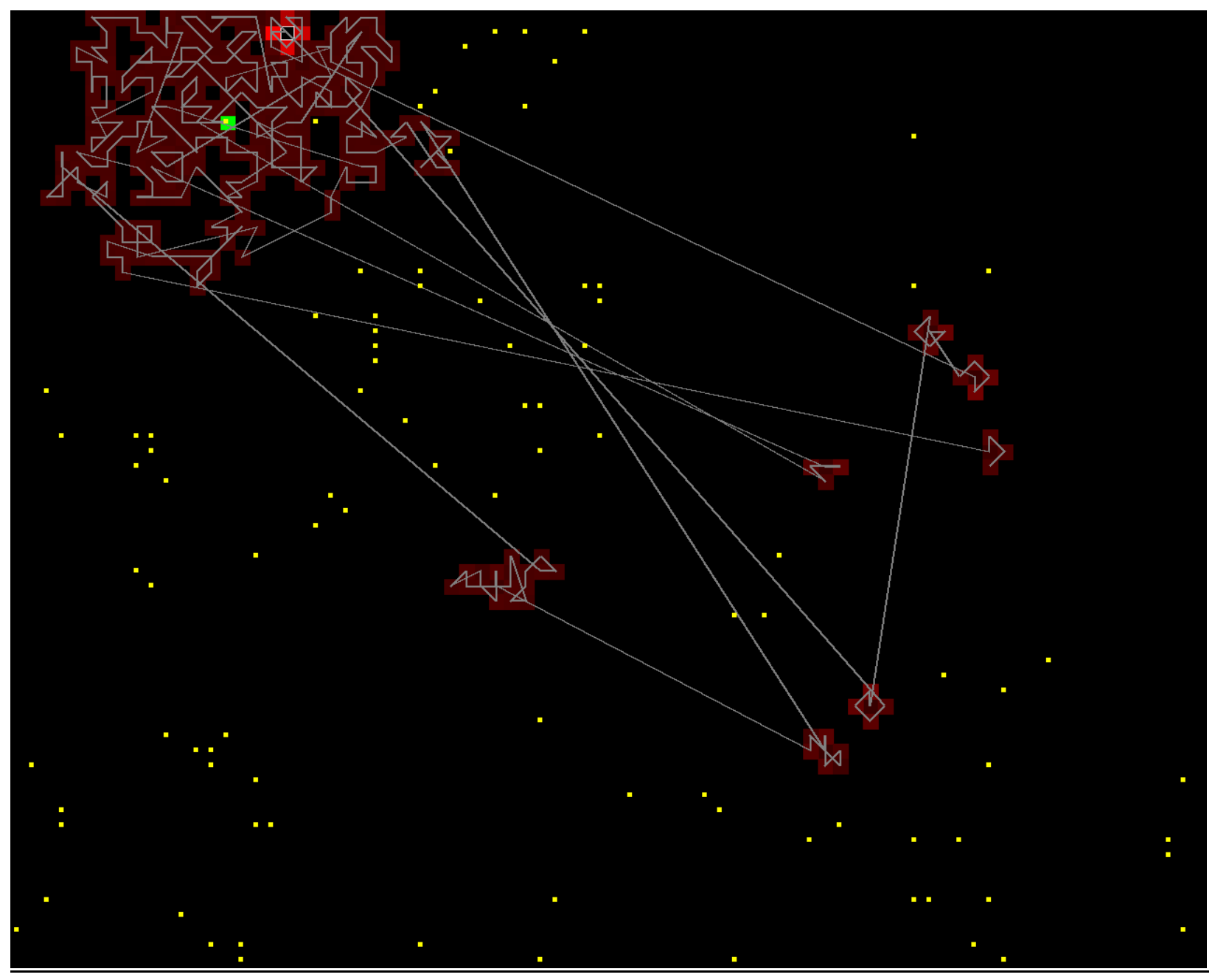

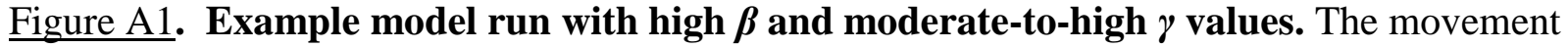
cost becomes overly strong and forces movements to mostly remain near one location. 


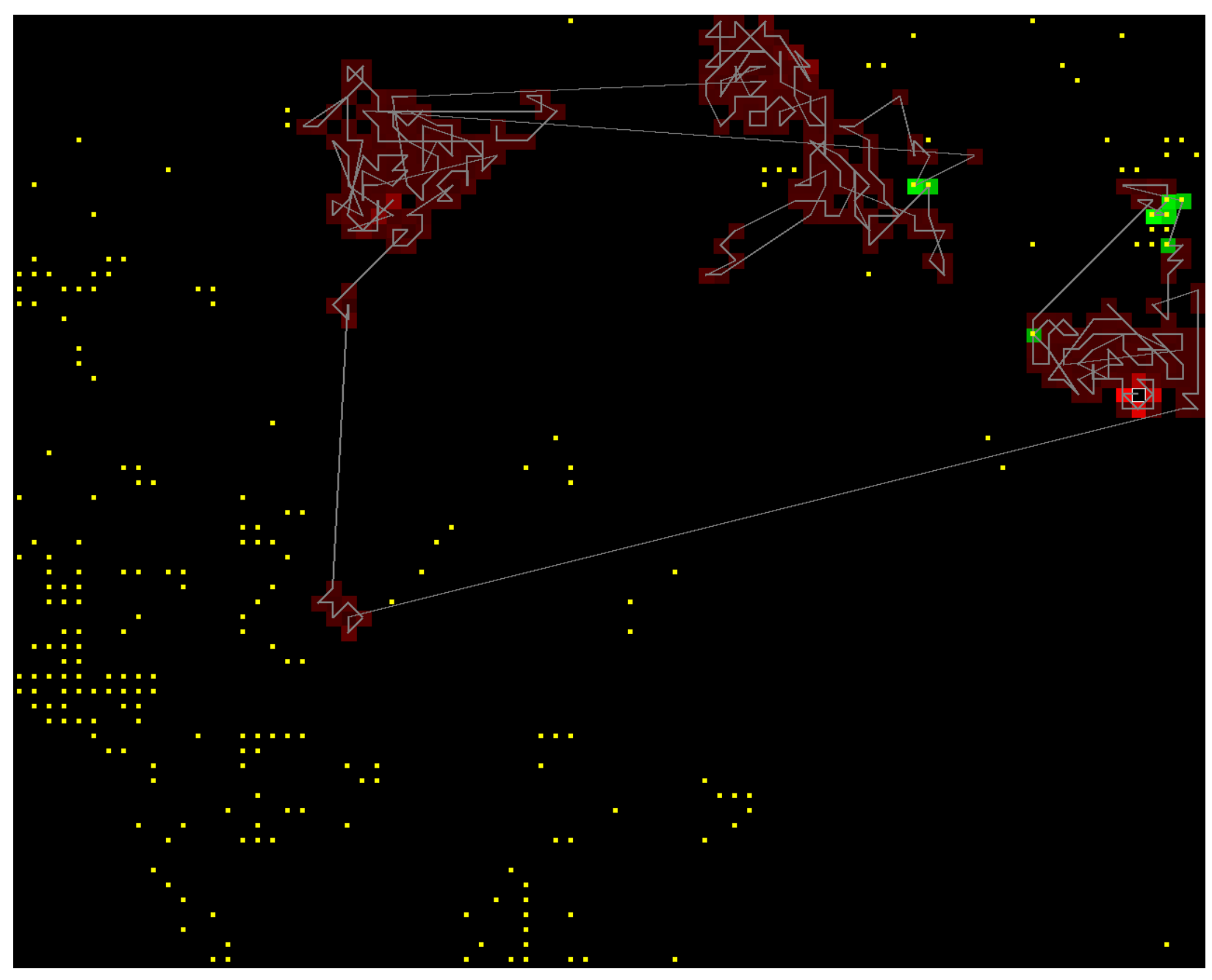

Figure A2. Example model run with high $\gamma$ and low-to-moderate $\beta$. High gain makes the probability distribution overly peaked, which causes the model to make movements among a small number of overly dense clusters. 


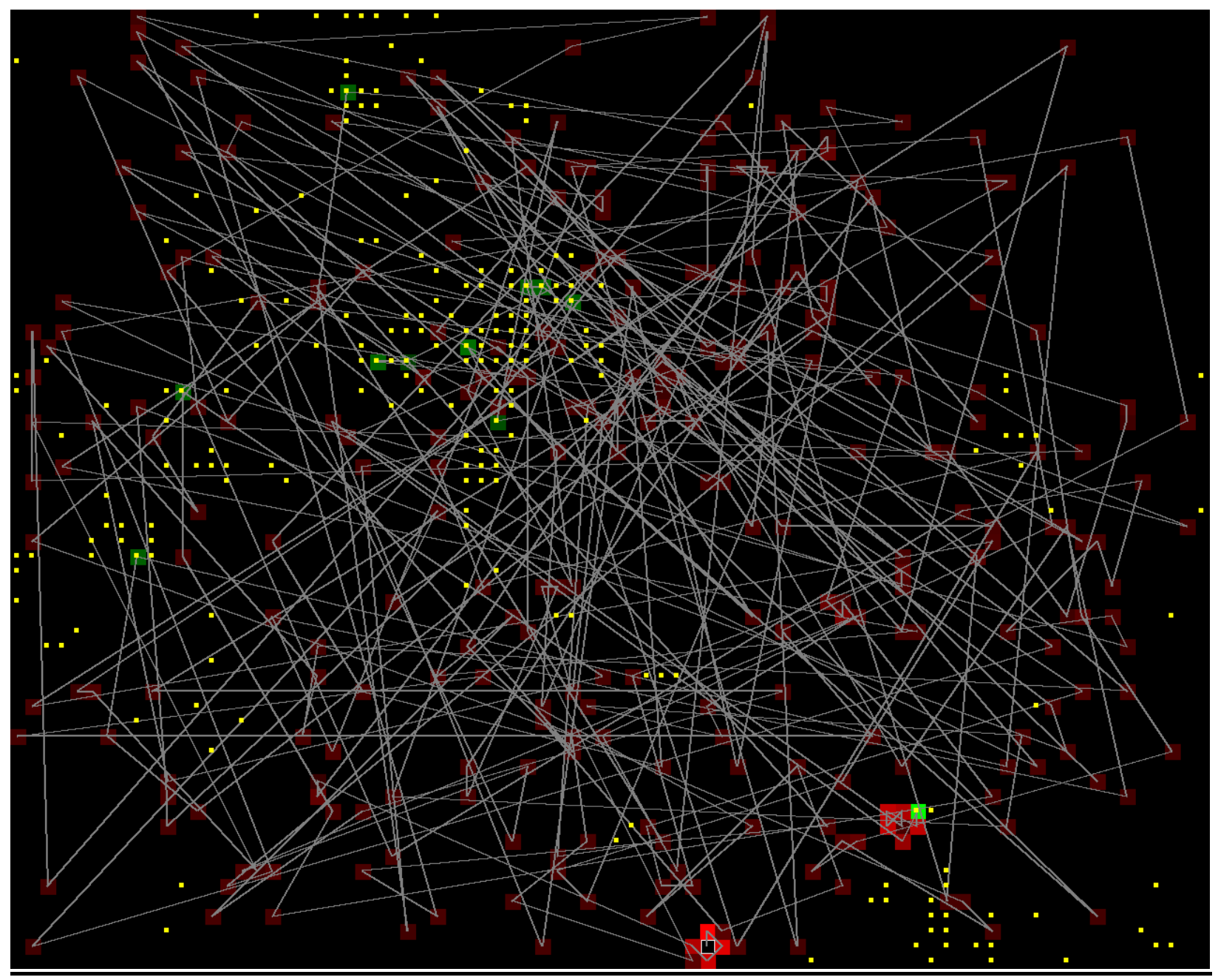

Figure A3. Example model run with low $\boldsymbol{\gamma}$ paired with any $\boldsymbol{\beta}$ values. Low gain makes the probability distribution too flat, which causes movements to become random and hence not sufficiently clustered. 